\title{
Erratum to: SuperTranscripts: a data driven reference for analysis and visualisation of transcriptomes
}

\author{
Nadia M. Davidson ${ }^{1,2^{*}}$, Anthony D. K. Hawkins ${ }^{1}$ and Alicia Oshlack ${ }^{1,2^{*}}$
}

\section{Erratum}

Upon publication of the original article [1], it was noted that data submitted by the authors was erroneously amended during proofing. The following section of the submitted manuscript was wrongfully amended in the Methods section, in the published article [1]:

The second line of the subsection "Read alignment" currently reads "-outSJfilterOverhangMin 1212 12" and should instead be "-outSJfilterOverhangMin 121212 12".

\section{Author details}

'Murdoch Children's Research Institute, Royal Children's Hospital, Melbourne,

Australia. ${ }^{2}$ School of BioSciences, University of Melbourne, Melbourne,

Australia.

Published online: 24 August 2017

\section{Reference}

1. Davidson NM, Hawkins AD, Oshlack A. SuperTranscripts: a data driven reference for analysis and visualisation of transcriptomes. Genome Biol. 2017;18(1):148.

Full list of author information is available at the end of the article 